\title{
Population-Based Registries are Important in Sarcoma: An Editorial Regarding "Incidence Patterns of Primary Bone Cancer in Taiwan (2003-2010)"
}

\author{
Benjamin J. Miller, MD, MS \\ Department of Orthopaedics and Rehabilitation, University of Iowa, Iowa City, IA
}

Ernest Codman is a well-known figure in musculoskeletal oncology. He is the source of common eponyms, such as 'Codman's triangle', the radiographic appearance of a periosteal reaction stimulated by an adjacent osseous malignancy, and 'Codman's tumor', more commonly referred to as a chondroblastoma. In addition, he was an early advocate of recording and analyzing 'end results' to truly assess the final outcomes of an intervention. ${ }^{1}$ In fact, with assistance from the family of a presumed sarcoma patient and the American College of Surgeons, he established the Registry of Bone Sarcoma in 1921 to make a record of any living patients cured of sarcoma, and to determine the means of attaining cure in those individuals. ${ }^{2}$ These simple goals manifested into the first concerted effort to document survival statistics, consolidate nomenclature, and determine the methods of cure in sarcoma. Although this initial effort was in a time before aggressive limb salvage, magnetic resonance imaging, modern chemotherapy, and the Internet, its basic goals and challenges continue to be relevant today.

The report by Hung et al ${ }^{3}$ underscores the importance of creating, maintaining, and reporting data generated by large population-based cancer registries. The rarity of sarcoma makes dedicated study extremely challenging, and single-institution reports of incidence, treatment, and results are unlikely to be robust enough to allow for definitive conclusions. Although large cancer-specific registries exist, such as the Surveillance, Epidemiology, and End Results Program Database in the US, they may not

(C) Society of Surgical Oncology 2014

First Received: 24 March 2014;

Published Online: 18 April 2014

B. J. Miller, MD, MS

e-mail: benjamin-j-miller@uiowa.edu be representative of populations elsewhere in the world. Specifically, Southeast Asia accounts for an inordinately large proportion of the world's population, and the description of sarcoma incidence in this region is necessary to define unique risk factors and allow for comparison to other locations. Furthermore, this study describes a homogenous population of Han Chinese (the largest ethnic group in the world) and further study may be performed in other places of similar ethnicity to explore the impact of environmental factors (exposure to carcinogens, diet, hormonal factors, etc.) while controlling for race.

It should be emphasized that the conclusions drawn from population-based registries are primarily hypothesis generating. Although many interesting observations spur thought and theory, it is really just a starting point. Curious or enticing findings should be used to design studies relevant to the real question: how can we improve patient care? In this study, for instance, the authors describe an increasing incidence of bone cancer over the study period. The underlying cause of this observation is not clear and could be due to many factors. Perhaps it is due to changes in dietary intake in the last decade, or exposure to different chemicals in the environment, or improvements in diagnostic techniques resulting in the perception of more cases, or changes in referral patterns, or simply noise in the data. In all likelihood, it is a combination of many factors, none of which can be clearly accepted or eliminated. Although these data provide for nothing other than speculation upon the competing explanations, the attention it draws to this issue may encourage this team or others to determine if there is a causal factor increasing the incidence of bone cancer, and if that factor can be addressed.

Investigators and readers should also beware that registries differ in terms of design, purpose, data collection, support, and scale. These differences can have a number of 
implications when trying to assess the quality of data from an individual registry. When the data source may not be familiar to the readership, reporting the basics of data collection help to justify the study conclusions. The research team should be familiar with the methods of data acquisition and report limitations that may skew conclusions (such as patient medical information gathered from administrative forms or billing records). In addition, readers should regard issues of data accuracy with polite skepticism. In this study, $30 \%$ of the 'other malignancy' category was not morphologically verified. The investigators very appropriately noted this limitation, and readers should regard conclusions from this group critically. In this we also observe that the challenges in diagnostic accuracy and reliable classification noted by Codman are far from resolved.

Data from large registries are often the best source to answer descriptive questions such as incidence, prevalence, survival, and risk stratification. An immediate application of this type of information is as a clinical aid when counselling patients. Previous investigations have concluded that patients respond well to specific numbers (incidence rates, survival statistics) even when the outlook is dismal. ${ }^{4,5}$

Finally, a paper such as this is an opportunity to consider the 'ideal' registry. As was Codman's experience, at a registry's inception lays an opportunity to define nomenclature. For sarcoma, as in most cancer, the key features to report for categorization include histologic diagnosis, location, grade, and stage. These are technically basic concepts, as they are the minimal amount of information required to reliably name a sarcoma, but are far from simple. To complicate this issue further, even basic nomenclature may change over time. Just as gross pathologic description evolved into sophisticated microscopic histology in the last century, future work may focus more on labeling genetic abnormalities, protein expression, epigenetic changes, or receptor status. Therefore, an ideal registry must be reliable, verifiable, reproducible, and universally accepted, while remaining fluid and able to adapt to changing paradigms and technology.

The primary goal of a registry, however, should be to improve the overall survival and quality of life for patients.
The knowledge gained from a registry, if unable to be applied to actual clinical questions, is akin to a missed opportunity. Clinically relevant data requires the registry to include information on treatment and outcomes. In sarcoma, the heterogeneity of histologic diagnosis is surpassed only by the heterogeneity of treatment, so the task of reporting chemotherapeutic modalities, final surgical margins, implants, oncologic and reconstructive complications, and functional and quality-of-life measures must be collected with maximal agreement among providers and administrators. This promises to be a recurring and important challenge moving forward.

To summarize, this study provides important information from a national sarcoma registry in Taiwan. Its strengths include capture of nearly all cases of bone sarcoma in a defined health system and reporting of new information specific to a common ethnicity. I hope to see this study followed by further investigations focused on diagnosis, treatment, and overall survival. With nearcomplete participation of the hospitals in Taiwan, this registry provides a remarkable opportunity to analyze delivery of care and investigate the efficacy of chemotherapeutic regimens and surgical intervention with minimal incomplete data or lost participants.

\section{REFERENCES}

1. McCarthy EF. The registry of bone sarcoma. A history. Iowa Orthop J. 1995;15:74-78.

2. Codman EA. The classic: registry of bone sarcoma. Part I: twentyfive criteria for establishing the diagnosis of osteogenic sarcoma. Part II: thirteen registered cases of "five year cures" analyzed according to these criteria. Clin Orthop Relat Res. 2009; 467(11):2771-2782.

3. Hung G-Y, Horng J-L, Yen H-J, Yen C-C, Chen W-M, Chen PC$\mathrm{H}$, et al. Incidence patterns of primary bone cancer in Taiwan (2003-2010): a population-based study. Ann Surg Oncol. doi:10. 1245/s10434-014-3697-3.

4. Mack JW, Wolfe J, Cook EF, Grier HE, Cleary PD, Weeks JC. Hope and prognostic disclosure. J Clin Oncol. 2007;25(35): $5636-5642$.

5. Mack JW, Wolfe J, Grier HE, Cleary PD, Weeks JC. Communication about prognosis between parents and physicians of children with cancer: parent preferences and the impact of prognostic information. J Clin Oncol. 2006;24(33):5265-5270. 